\title{
Transmission Imaging With Axially Overlapping Cone-Beams
}

\author{
B. Feng, Member, IEEE, J. A. Fessler, senior Member, IEEE, P. H. Pretorius, Associate Member, \\ IEEE, G. Boening, Member, IEEE, R. D. Beach, Senior Member, IEEE, G. L. Zeng, Senior Member, \\ IEEE, M. A. King, Senior Member, IEEE
}

\begin{abstract}
We have shown that cone-beam transmission imaging of medium-energy photons that penetrate the parallel-hole collimators can be used to rapidly estimate attenuation maps for use in reconstruction of cardiac SPECT images. Such a transmission imaging geometry offers the advantages of eliminating the need to mechanically move the point-sources during imaging, and minimizes cross-talk between emission and transmission imaging. The axial extent over which artifact-free attenuation maps can be reconstructed is limited by the conebeam geometry and source collimation. We investigated irradiation of a single head by multiple point-sources such that their asymmetric cone-beam fields overlap in the axial direction as a method of extending the axial coverage of the patient. This study reports on testing of a penalized-likelihood algorithm for transmission reconstruction of overlapping cone-beams. This algorithm was evaluated through MCAT simulations and applied to transmission measurements of an anthropomorphic phantom. The experimental work consisted of performing a series of flood and transmission measurements on the anthropomorphic phantom with shifted axial locations of point-sources. We summed the projection data from individual measurements to simulate the projection data for a multiple point-source system. With the proposed penalized-likelihood algorithm, the full axial extent $(20.5 \mathrm{~cm})$ of the anthropomorphic phantom was reconstructed for the overlapping cone-beam geometry with 2 point-sources per camera head.
\end{abstract}

\section{INTRODUCTION}

$\mathrm{W}$ E have shown that cone-beam transmission imaging of medium-energy photons that penetrate the parallel-hole collimators can be used to rapidly estimate attenuation maps for use in reconstruction of cardiac SPECT images [1-4]. Such a transmission imaging geometry (Fig. 1, 2) offers the advantages of eliminating the need to mechanically move the

Manuscript received October 19, 2004. This work was supported by grant HL50349 from the National Heart, Lung, and Blood Institute.

B. Feng, P. H. Pretorius, R. D. Beach, and G. Boening and M. A. King are with the Department of Radiology, University of Massachusetts Medical School, Worcester, MA 01655. (telephone: 508-856-8795, e-mail: Bing.Feng@umassmed.edu).

J. A. Fessler is with the Department of Electrical Engineering and Computer Science, University of Michigan, Ann Arbor, MI 48109. (e-mail: fessler@umich.edu).

G. L. Zeng is with the Department of Radiology, University of Utah, Salt Lake City, UT 84108. (e-mail: larry@ucair.med.utah.edu). point-sources during imaging, and minimizes cross-talk between emission and transmission imaging. With the help of Philips Medical Systems, we modified the Beacon transmission device [5] on the IRIX SPECT system in our clinic to perform dual-planar asymmetric cone-beam transmission imaging. This was achieved by positioning each of two point-sources at a fixed axial location before the scan and keeping it fixed during the scan (Fig. 1). Using the conebeam geometry, transmission acquisition can be performed within 40 second on the IRIX system (Fig. 3). The axial extent over which artifact-free attenuation maps can be reconstructed is limited $(\sim 16 \mathrm{~cm})$ by the cone-beam geometry and source collimation. Though this axial extent is adequate for cardiac SPECT, it is not adequate for other applications like boneSPECT. We investigated irradiation of a single head by multiple point-sources such that their asymmetric cone-beam fields overlap in the axial direction as a method of extending the axial coverage of the patient. This study reports on testing of a penalized-likelihood algorithm for transmission reconstruction of overlapping cone-beams.
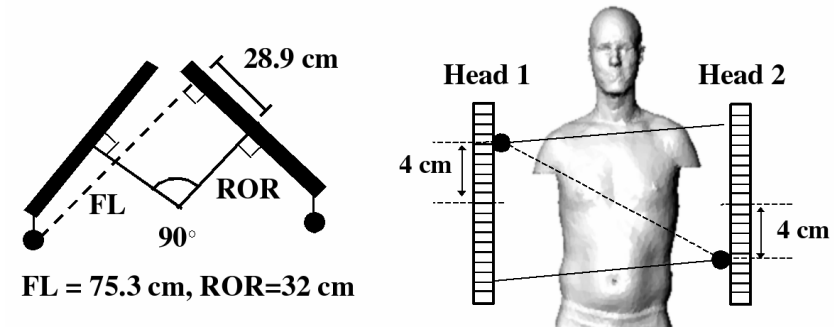

Fig. 1. The trans-axial (left) and axial (right) views of the non-overlapping cone-beam geometry achieved on the Beacon system.

\section{METHODS AND RESUltS}

\section{A. Evaluation of the OSTR Algorithm for Overlapping Cone-Beams through MCAT Simulations}

The OSTR (Ordered Subset Transmission Reconstruction) algorithm [6] models the Poisson noise in the transmission imaging. A penalty term can be easily incorporated into the OSTR algorithm to penalize the roughness of the attenuation map [6]. The penalized OSTR algorithm was shown to outperform the non-penalized algorithms in reconstructing the extremely low-count $(\sim 1 \mathrm{M})$ transmission data acquired in a 40 -second scan [3]. The reconstruction algorithm adopted in 
this study is the penalized OSTR algorithm tailored to the overlapping-beam geometry [7]. The roughness penalty included is of Huber type [8], which preserves the edge feature.

The proposed overlapping cone-beam geometry (Fig. 4) consists of a total of 4 Ba-133 point-sources (two per head), which are deployed axially at $-8,-2,2,8 \mathrm{~cm}$, respectively. The gantry angle tested in this study is 90 degrees between two heads hosting the point-sources. This multiple cone-beam system can be achieved physically by adding more Ba-133 point-sources to the Beacon transmission system, or simulated as in this study by performing multiple measurements of the same object with the point-sources at various axial locations on the two point-source non-overlapping geometry (the modified Beacon, Fig. 1).

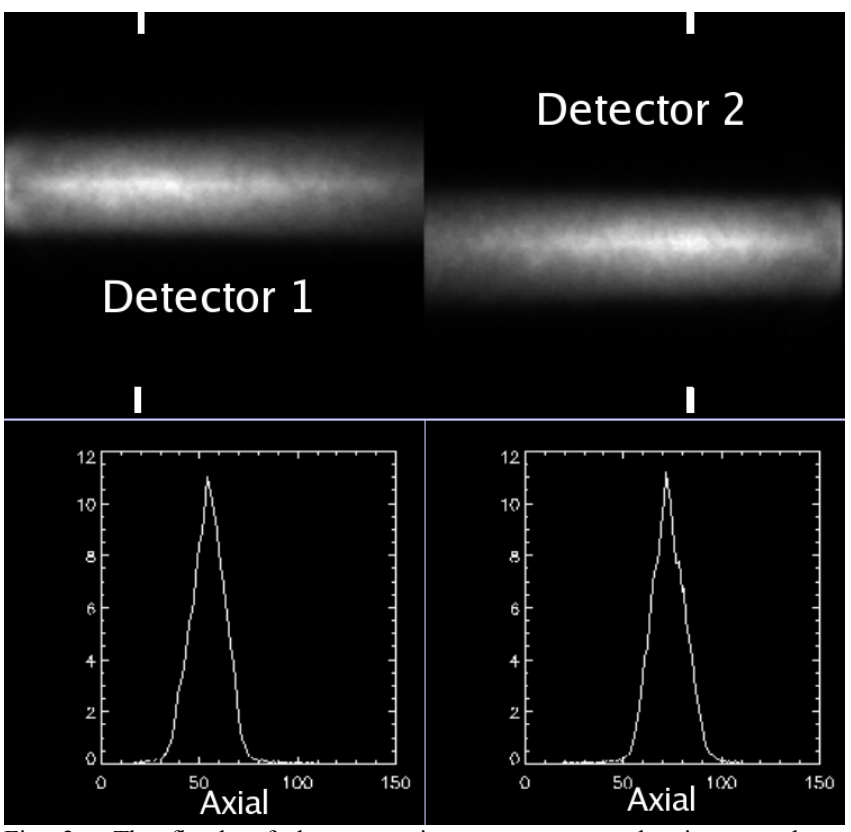

Fig. 2. The floods of the two point-source non-overlapping cone-beam geometry (the modified Beacon). The axial profile on each detector is also plotted.

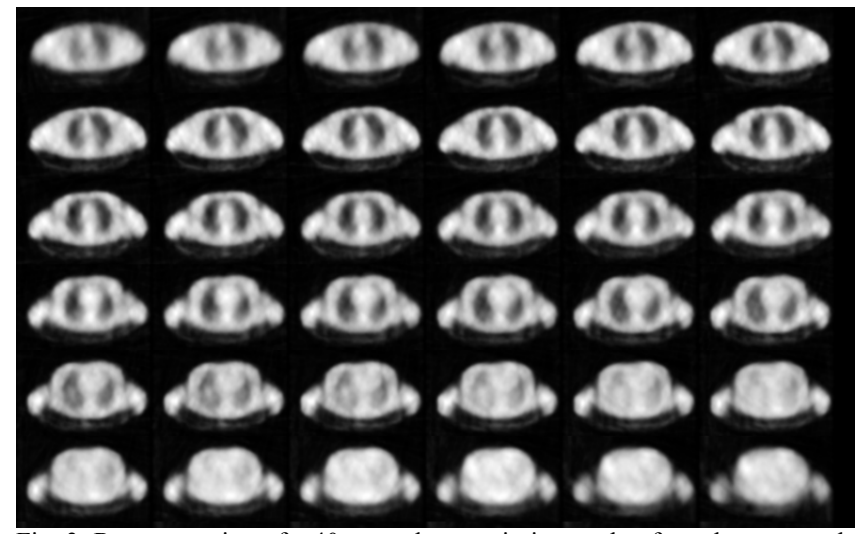

Fig. 3. Reconstruction of a 40 second transmission study of a volunteer on the modified Beacon system. The axial offset between two point-sources is $8 \mathrm{~cm}$.
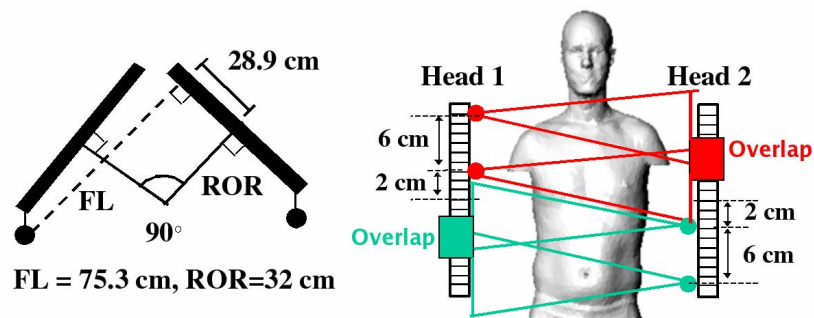

Fig. 4. The transaxial (left) and axial (right) views of the overlapping conebeam geometry investigated. FL - the focal length, ROR - the radius of rotation. The geometric parameters are adopted from the IRIX Beacon transmission system.
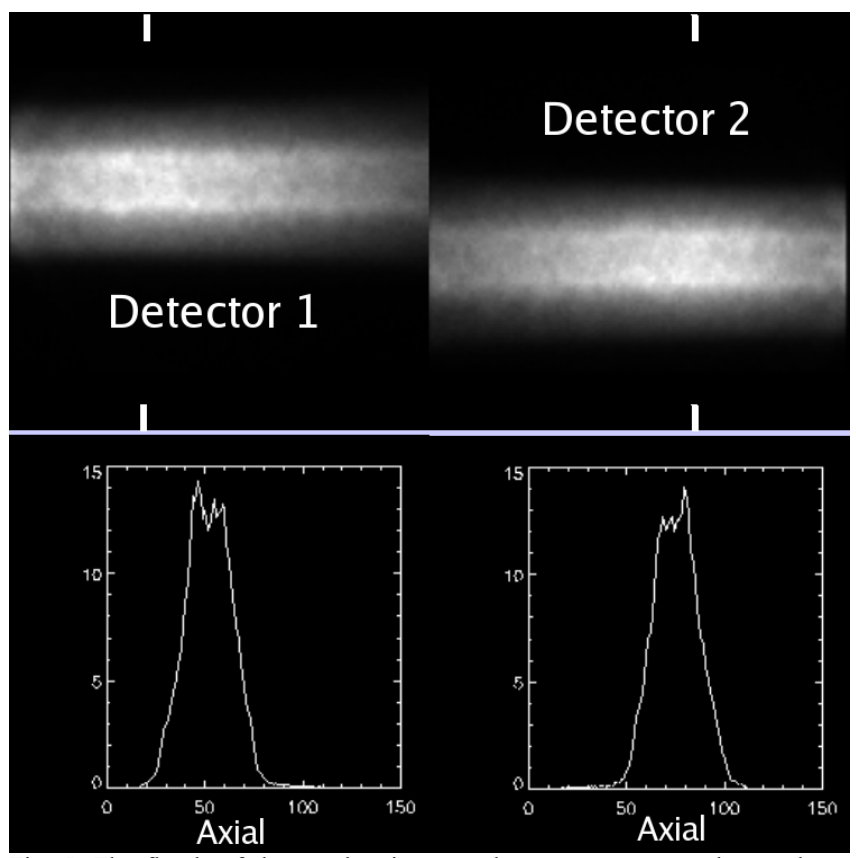

Fig. 5. The floods of the overlapping cone-beam geometry as shown above. The axial profile on each detector is also plotted. Note that the floods from two point-sources severely overlap on each detector.

The flood for each point-source was measured on the modified Beacon using a 4-minute blank scan. The energy window was centered at $356 \mathrm{keV}$ and with a $15 \%$ window width. The floods of the two point-sources irradiating the same detector severely overlapped (Fig. 5). Using a numerical projector for the overlapping cone-beam geometry and the true floods, the transmission projection data for a 360-degree acquisition of an MCAT phantom were simulated by raytracing. The numerical projector had been validated by simulations of analytical spherical phantoms. Using 20 iterations of the OSTR, the reconstruction from the simulated transmission projections shows excellent agreement with the original MCAT phantom (Fig. 6).

As a comparison, a simulation of the MCAT was also performed with the non-overlapping cone-beam geometry as shown in Figure 1. The simulated projection data were also reconstructed with 20 iterations of the OSTR. As expected, the axial coverage of the overlapping cone-beam geometry was found to be about $7 \mathrm{~cm}$ wider than the axial coverage of the non-overlapping cone-beam geometry (Fig. 7). The axial extent of the MCAT phantom is $50 \mathrm{~cm}$. Once the OSTR 
algorithm for the overlapping cone-beams had been validated, it was applied to the transmission measurements of an anthropomorphic phantom as described in the next section.

\section{B. Transmission Measurements of an Anthropomorphic Phantom and Reconstruction with the OSTR algorithm}

We performed a series of flood (4 minutes) and transmission (2 minutes) measurements on the Data Spectrum Anthropomorphic phantom (which is of $20.5 \mathrm{~cm}$ axial extent) with axial locations of point-sources shifted by $-8,-2,2,8 \mathrm{~cm}$ relative to the center of the field of view of the camera heads. We summed the projection data from individual measurements to simulate the projection data from the overlapping conebeam geometry shown in Figure 4 . The reconstruction using 20 iterations of the OSTR algorithm is shown in Figure 8.

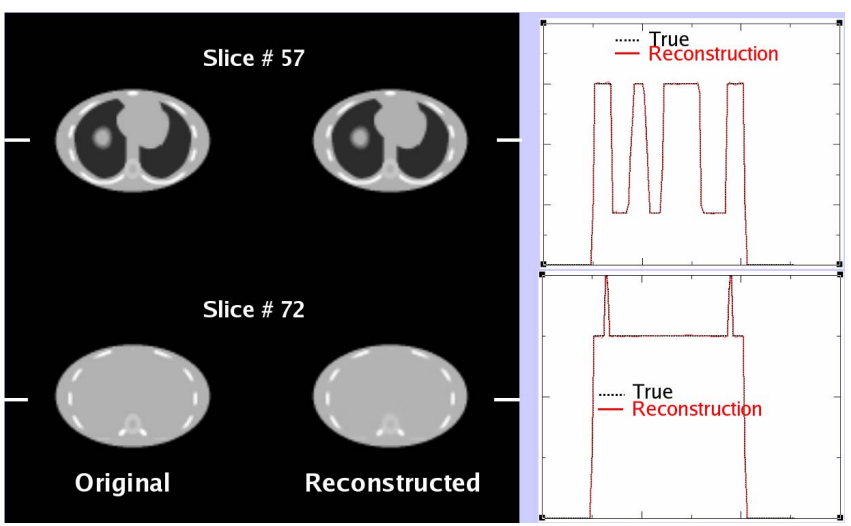

Fig. 6. (Left) Two slices of the original MCAT phantom and the reconstruction from 20 iterations of OSTR algorithm. (Right) Line profiles through the center on both slices.

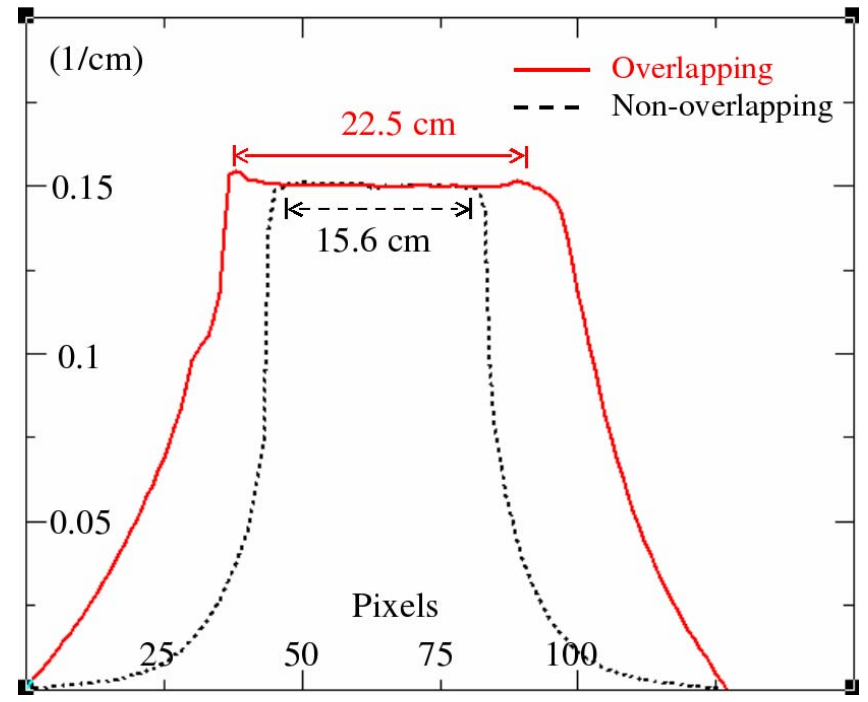

Fig. 7. The axial profiles through attenuation maps reconstructed for the nonoverlapping (Fig. 1) and overlapping geometries (Fig. 4). The axial coverage of the overlapping geometry is about $22.5 \mathrm{~cm}$.

As a comparison, the reconstruction for the non-overlapping cone-beam geometry is shown in Figure 9. The improved axial coverage with the overlapping cone-beam geometry is evident. Note the presence of the knobbed screws and caps to seal the ports for adding water and activity to the various phantom compartments are seen in the slices at the top and bottom of Figure 8 , indicating the axial coverage of the system was greater than the $20.5 \mathrm{~cm}$ extent of the phantom.

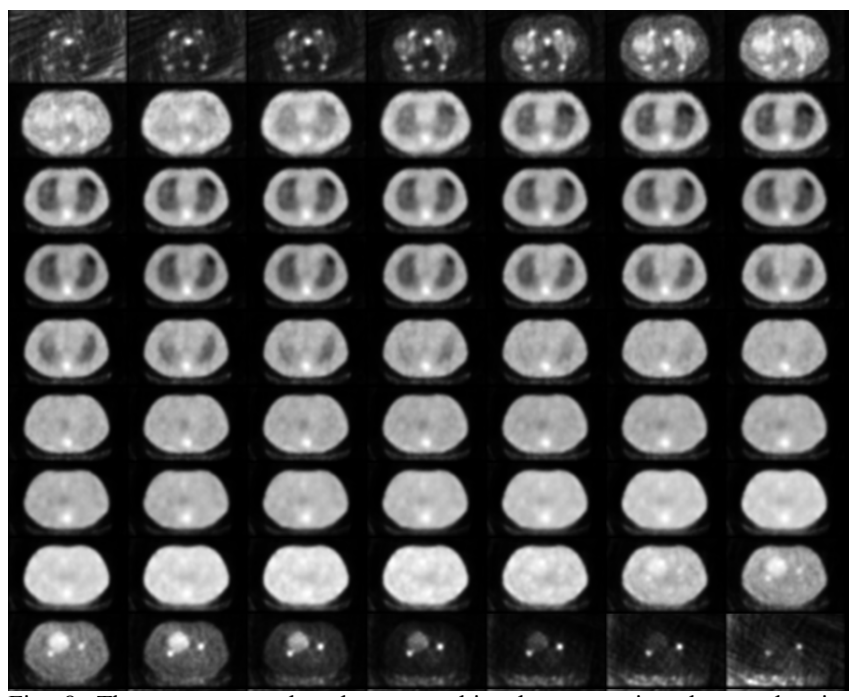

Fig. 8. The reconstructed anthropomorphic phantom using the overlapping geometry shown in Fig. 4. The acquisition is 2 minutes.

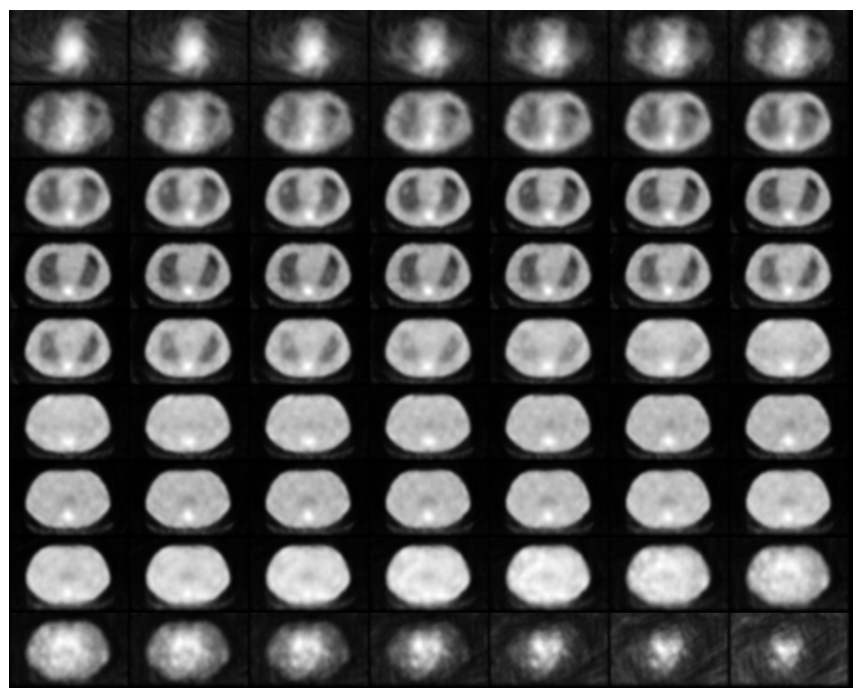

Fig. 9. The reconstructed anthropomorphic phantom using the non-overlapping geometry shown in Fig. 1. The acquisition is 2 minutes.

\section{DISCUSSION}

The investigated overlapping cone-beam geometry (Fig. 2) can provide about $22.5 \mathrm{~cm}$ axial coverage in the MCAT simulation (Fig. 7). This axial extent is adequate for imaging the entire anthropomorphic phantom (Fig. 8). The floods of two point-sources overlap severely, which is ideal for evaluating the reconstruction algorithm. Based on the flood pattern of a single point-source, we optimized the axial offset between the two point-sources for the non-overlapping conebeam system [2]. The optimal offset is about $7 \mathrm{~cm}$. Imagine that we couple two non-overlapping systems together and with a $7 \mathrm{~cm}$ offset between them. The resulted arrangement of the 
point-sources is plotted in Figure 10. The floods (Fig. 11) are less severely overlapped, which results in fewer iterations of the OSTR needed for reconstruction of the slices. Using the MCAT simulation as before and 10 iterations of the OSTR, we found out the axial coverage of this geometry is about $28 \mathrm{~cm}$ (Fig. 12).

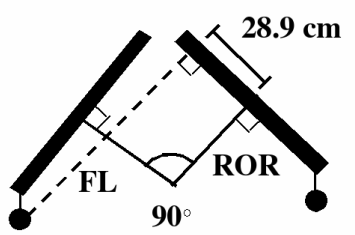

$F L=75.3 \mathrm{~cm}, \mathrm{ROR}=32 \mathrm{~cm}$

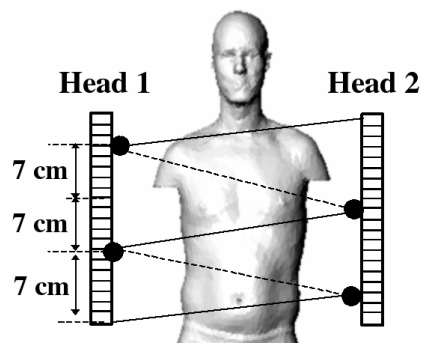

Fig. 10. The transaxial (left) and axial (right) views of an alternative arrangement of point-sources that can provide wider axial coverage than the geometry shown in Fig. 4.

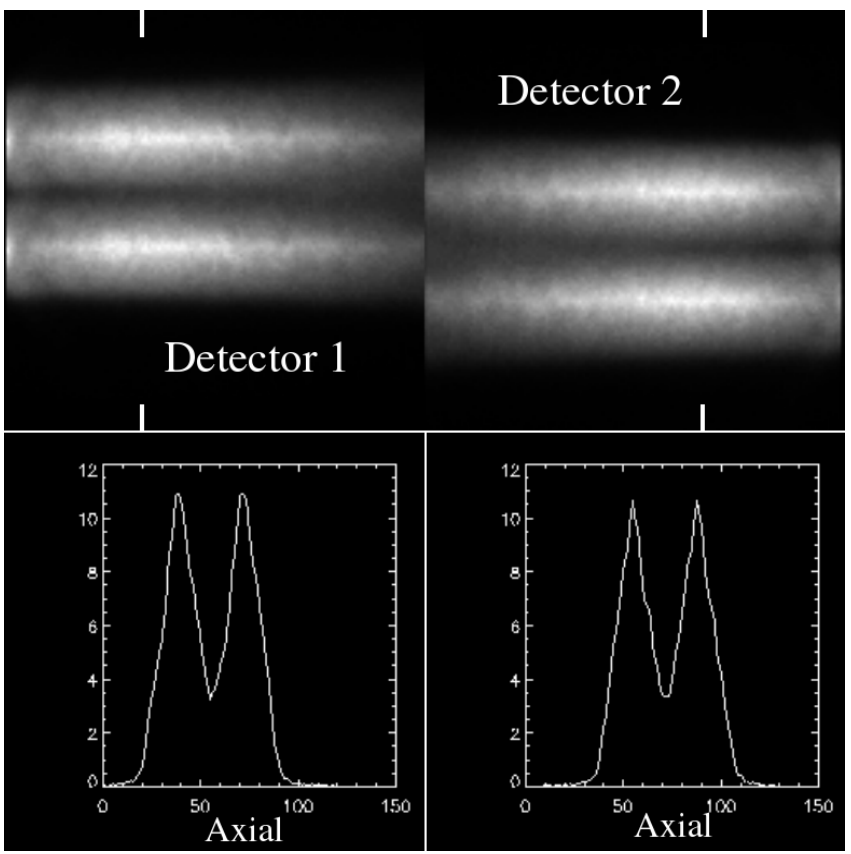

Fig. 11. The summed floods on each detector of the overlapping cone-beam system shown in Fig. 10.

As previous work [9], we investigated the feasibility of adapting the asymmetric cone-beam transmission imaging geometry to imaging systems which cannot rotate 360 degrees. For those systems with limited gantry rotation, a denser array of point-sources (Fig. 13) may help reducing the artifacts caused by the limited angle reconstruction, for such a denser array approximates more to the fan-beam geometry. We performed the MCAT simulation with the geometry shown in Figure 13, and found that the proposed OSTR algorithm provided accurate reconstruction but took more iterations (about 40) for accurate reconstruction.

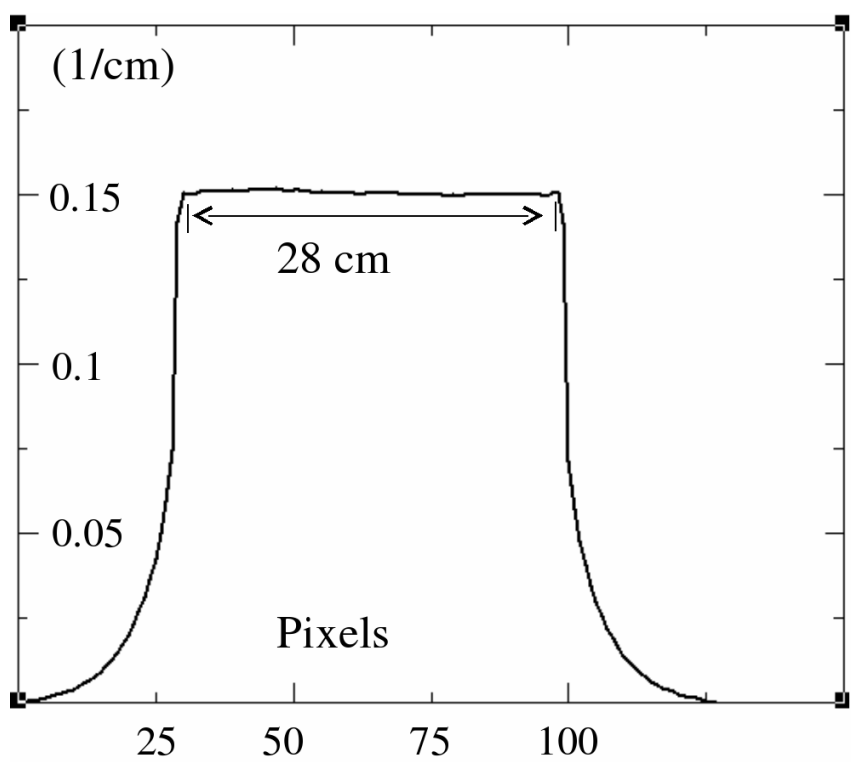

Fig. 12. The axial profile through the center of the reconstructed MCAT phantom using the overlapping cone-beam geometry shown in Fig. 10. The axial coverage is approximately $28 \mathrm{~cm}$.
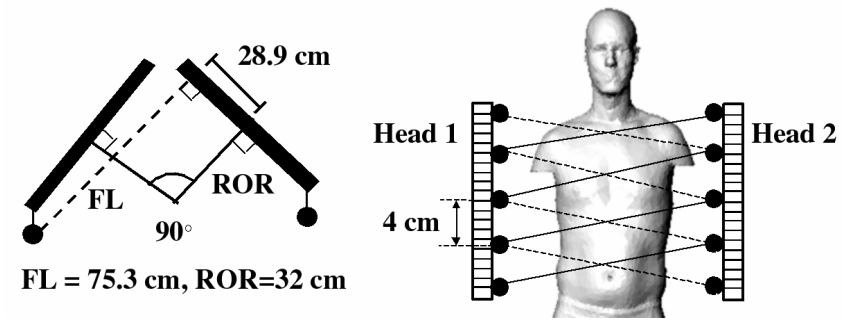

Fig. 13. The transaxial (left) and axial (right) views of 10 point-source system.

\section{CONCLUSION}

Using simulations of the MCAT phantom, we validated the OSTR algorithm tailored to the overlapping cone-beam geometry and investigated the axial coverage for several conebeam geometries. We found out that the overlapping conebeam geometry can provided substantial increase on the axial coverage over the non-overlapping cone-beam system. By applying the algorithm to the transmission measurement of the anthropomorphic phantom, good slices were reconstructed through the whole anthropomorphic phantom. We concluded that the axially overlapping cone-beam geometry might be a choice for the design of a fast transmission imaging system which covers about $28 \mathrm{~cm}$ of the thorax.

\section{REFERENCE}

[1] G. L. Zeng, G. T. Gullberg, and P. E. Christian, "Asymmetric ConeBeam Transmission Tomography," IEEE Trans Nucl Sci, vol 48, pp. 117-124, Feb 2002.

[2] B. Feng, M. A. King, G. L. Zeng, P. H. Pretorius, P. P. Bruyant, R. D. Beach, G. Boening, G. Jarkewicz, S. Cochoff, and D. Gagnon, "The Estimation of Attenuation Maps for Cardiac-SPECT Using Cone-Beam Imaging of High-Energy Photons Through Parallel-Hole Collimators," IEEE Trans. Nucl. Sci., in press.

[3] B. Feng, J. A. Fessler, G. Boening, R. D. Beach, M. A. King, "Penalized Likelihood Reconstruction for Dual-Planar Cone-Beam Transmission Imaging," [abstract], J. Nucl. Med., vol. 45, 104P, May 2004. 
[4] B. Feng, H. P. Pretorius, P. P. Bruyant, G. Boening, R. D. Beach, J. A. Fessler, M. A. King, "Impact on Cardiac Reconstruction of Using Attenuation Maps Reconstructed with Less Than Complete Rotation Acquired by Asymmetric Cone-Beam Transmission Imaging," [abstract], J. Nucl. Med., vol. 45, 158P, May 2004.

[5] D. Gagnon, C. H. Tung, G. L. Zeng, W. Hawkins, "Design and early testing of a new medium-energy transmission device for attenuation correction in SPECT and PET," Conference Record of the 1999 IEEE Nuclear Science Symposium, Seattle, WA, Oct, 24-30.
[6] H. Erdoğan and J. A. Fessler, "Ordered Subsets Algorithms for Transmission Tomography," Phys. Med. Biol., vol. 44, pp. 2835-51, 1999.

[7] D. F. Yu, J. A. Fessler, and E. P. Ficaro, "Maximum Likelihood Transmission Image Reconstruction for Overlapping Transmission Beams," IEEE Tr. Med. Im., vol. 19, pp. 1094-1105, 2000.

[8] P. J. Huber, Robust Statistics, Wiley, New York, 1981.

[9] B. Feng, P. H. Pretorius, P. P. Bruyant, G. Boening, R. D. Beach, H. C. Gifford, J. A. Fessler, M. A. King, "Body-and-Lungs-Outlines PenalizedLikelihood Reconstruction of Asymmetric Cone-Beam Transmission Data Acquired With Less Than Complete Gantry Rotation," [abstract] American Society of Nuclear Cardiology Annual Meeting, Sep. 30-Oct. 3,2004 . 